PROCEEDINGS OF THE

AMERICAN MATHEMATICAL SOCIETY

Volume 130, Number 10, Pages 3091-3096

S 0002-9939(02)06402-X

Article electronically published on March 13, 2002

\title{
ON CLASSES OF MAPS WHICH PRESERVE FINITISTICNESS
}

\author{
AKIRA KOYAMA AND MANUEL A. MORON \\ (Communicated by Alan Dow)
}

\begin{abstract}
We shall prove the following: (1) Let $r: X \rightarrow Y$ be a refinable map between paracompact spaces. Then $X$ is finitistic if and only if $Y$ is finitistic. (2) Let $f: X \rightarrow Y$ be a hereditary shape equivalence between metric spaces. Then if $X$ is finitistic, $Y$ is finitistic.
\end{abstract}

\section{INTRODUCTION}

To extend the classical cohomological methods in the study of group actions on compact Hausdorff spaces or finite-dimensional paracompact spaces Swan $\underline{\mathrm{Sw}}$ introduced the concept of finitistic spaces and obtained a Smith-type fixed point theorem. Typical results on group actions for finitistic spaces may be found in $[\mathrm{B}$, De-Si ${ }_{1}$, De-Si-S] and [De-T].

Definition. A space $X$ is said to be finitistic if every open cover of $X$ has an open refinement of finite order.

By the definition, the class of paracompact, finitistic spaces may be considered the natural one combining both compact and finite-dimensional paracompact spaces. Recently from the dimension-theoretical viewpoint several authors investigated finitistic spaces as a kind of infinite-dimensional spaces (cf. [De-P], $\left[\mathrm{De}_{-} \mathrm{Si}_{2}\right.$, Dy-M-S, [H1 and [H2]). Moreover, Rubin and Schapiro [Ru-Sc2 succeeded to show that the class of paracompact, finitistic spaces has a nice role in cohomological dimension theory. Namely

Theorem (Rubin and Schapiro). Suppose that $X$ is a paracompact, finitistic space and $G$ is a finitely generated abelian group. Then:

(1) $\operatorname{dim}_{G} \beta X=\operatorname{dim}_{G} X$, where $\beta X$ is the Stone-Čech compactification of $X$,

(2) if $X$ is separable and metrizable, then $X$ has a metrizable compactification $k X$ with $\operatorname{dim}_{G} k X=\operatorname{dim}_{G} X$.

On the other hand, since refinable maps were originally introduced by Ford and Rogers [F-R] to study continuum theory, many authors have found dimensiontheoretical properties of refinable maps (cf. [A], C-V], G-Ro, $\mathrm{Ka}], \mathrm{Ka}-\mathrm{Ko}$, Ko1, Ko2 and Ko-Sh]). For a refinable map between metric spaces the first

Received by the editors December 12, 2000 and, in revised form, April 24, 2001.

2000 Mathematics Subject Classification. Primary 55M10, 54F45.

Key words and phrases. Finitistic spaces, refinable maps, c-refinable maps, hereditary shape equivalences, extension dimension, cohomological dimension.

This work was started when the first author visited Departmento de Geometria y Topologia, Facultad de Ciencias Matematicas, Universidad Complutense de Madrid.

(C)2002 American Mathematical Society 
author [Ko2] showed that $\operatorname{dim} X=\operatorname{dim} Y$ and in [Ko-Sh] that $\operatorname{dim}_{G} X=\operatorname{dim}_{G} Y$ for any finitely generated abelian group $G$. In case $r$ is c-refinable, we also have that $K \in A E(X)$ if and only if $K \in A E(Y)$ for any simplicial complex $K$ (see Ko2, Theorem 1). Recently Chigogidze and Valov [C-V] generalized those results. Namely, if $f: X \rightarrow Y$ is a refinable map between metric spaces $X$ and $Y$, and $K$ is a $C W$-complex, then $e-\operatorname{dim} X \leq K$ if and only if $e-\operatorname{dim} Y \leq K$. Note that this result is true for a refinable map between compact Hausdorff spaces.

Similarly, by the definition, we can easily see that if $f: X \rightarrow Y$ is a hereditary shape equivalence between metric spaces $X$ and $Y$, and $e$ - $\operatorname{dim} X \leq K$, where $K$ is a $C W$-complex, then $e-\operatorname{dim} Y \leq K$. Therefore hereditary shape equivalences preserve dimension and cohomological dimension. Moreover, they preserve property $\mathrm{C}$ (see [A]) and small weak infinite-dimensionality (see [Mi]). Recently Dijkstra Di] and Dijkstra and Mogilski Di-Mo] gave an interesting example and results about small transfinite inductive dimension and countable dimensionality. Namely, we can say that hereditary shape equivalences have interesting dimension-theoretical properties.

In this paper, first, we shall show that if $r: X \rightarrow Y$ is a refinable map between paracompact spaces, then $X$ is finitistic if and only $Y$ is finitistic. We shall note remarks on the extension property of finitistic spaces. Next, we shall show that if $r: X \rightarrow Y$ is a hereditary shape equivalence between metric spaces and $X$ is finitistic, $Y$ is also finitistic. Note that Dydak, Mishra and Shukla Dy-M-S discussed several mapping theorems for finitistic spaces.

All spaces considered in this paper are assumed to be normal and maps are continuous.

\section{REFINABLE MAPS AND FINITISTICNESS}

Let $\mathcal{P}$ be a class of (not necessarily compact) polyhedra. A space $X$ is said to be $\mathcal{P}$-like if for every locally finite open cover $\mathcal{U}$ of $X$ there exists a $\mathcal{U}$-map $\varphi: X \rightarrow P \in \mathcal{P}$, here a $\mathcal{U}$-map means that each point $z \in P$ has a neighborhood $O_{z}$ such that $\varphi^{-1}\left(O_{z}\right)$ is contained in an element of $\mathcal{U}$. It is well-known that a paracompact space $X$ has $\operatorname{dim} X \leq n$ if and only if $X$ is $\mathcal{P}_{n}$-like, where $\mathcal{P}_{n}$ is the class of all polyhedra of dimension $\leq n$. In a similar way we can find a similar characterization of paracompact, finitistic spaces as follows:

Proposition 2.1. Let $\mathcal{P}_{f}$ be the class of all finite-dimensional polyhedra. Then a paracompact space $X$ is finitistic if and only if $X$ is $\mathcal{P}_{f}$-like.

A surjective map $r: X \rightarrow Y$ is called refinable if for any open cover $\mathcal{U}$ of $X$ and any open cover $\mathcal{V}$ of $Y$ there exists a surjective $\mathcal{U}$-map $f: X \rightarrow Y$ such that $r$ and $f$ are $\mathcal{V}$-close, that is, for every point $x \in X$ there is an element of $\mathcal{V}$ containing both $r(x)$ and $f(x)$, and shortly denoted by $d(r, f) \leq \mathcal{V}$. The map $f$ is called a $(\mathcal{U}, \mathcal{V})$-refinement of $r$. When there exists a closed $(\mathcal{U}, \mathcal{V})$-refinement of $r$, we say that $r$ is c-refinable.

Theorem 2.2. Let $\mathcal{P}$ be a class of polyhedra and let $r: X \rightarrow Y$ be a refinable map between paracompact spaces. Then $X$ is $\mathcal{P}$-like if and only if $Y$ is $\mathcal{P}$-like.

Proof. First we suppose that $X$ is $\mathcal{P}$-like. For a given open cover $\mathcal{V}$ of $Y$, let us take a locally finite open refinement $\mathcal{U}$ of $r^{-1}(\mathcal{V})$. By the definition there are a $P \in \mathcal{P}$ and a $\mathcal{U}$ - map $\varphi: X \rightarrow P$. Then there is a locally finite open cover $\tilde{\mathcal{U}}$ of $P$ such 
that

$$
\varphi^{-1}(\operatorname{St}(\tilde{\mathcal{U}})) \prec r^{-1}(\mathcal{V}) ;
$$

here $\operatorname{St}(\tilde{\mathcal{U}})=\{\operatorname{St}(\tilde{U}, \tilde{\mathcal{U}}) \mid \tilde{U} \in \tilde{\mathcal{U}}\}$ and $\operatorname{St}(\tilde{U}, \tilde{\mathcal{U}})=\cup\left\{\tilde{U}_{*} \in \mathcal{U} \mid \tilde{U} \cap \tilde{U}_{*} \neq \emptyset\right\}$. We choose a sufficiently small triangulation $T$ of $P$ such that

$$
\mathcal{T}=\left\{\operatorname{st}(v, T) \mid v \in T^{(0)}\right\} \prec \tilde{\mathcal{U}} .
$$

Then we can take a $\left(\varphi^{-1}(\mathcal{T}), \mathcal{V}\right)$-refinement $f: X \rightarrow Y$ of $r$. Since $f$ is a $\varphi^{-1}(\mathcal{T})$ map, there exists a locally finite open cover $\mathcal{W}$ of $\mathrm{Y}$ such that

$$
\begin{gathered}
\mathcal{W} \prec \mathcal{V} \text { and } \\
f^{-1}(\mathcal{W}) \prec \varphi^{-1}(\mathcal{T}) .
\end{gathered}
$$

Moreover we may assume that the cover $\mathcal{W}$ is given by cozero sets of a partition of unity $\left\{\xi_{W}\right\}_{W \in \mathcal{W}}$. Hence we can define the map $\eta: Y \rightarrow N(\mathcal{W})$ of $Y$ to the nerve $N(\mathcal{W})$ of $\mathcal{W}$ by $\eta(y)=\sum_{W \in \mathcal{W}} \xi_{W}(y) \cdot W$.

For each $W \in \mathcal{W}$, by (4), there exists a $v_{W} \in T^{(0)}$ such that

$$
f^{-1}(W) \subset \varphi^{-1}\left(s t\left(v_{W}, T\right)\right) .
$$

For a finite subset $\left\{W_{0}, \cdots, W_{n}\right\}$ of $\mathcal{W}$, if $\bigcap_{i=0}^{n} W_{i} \neq \emptyset$, by $(5), \bigcap_{i=0}^{n} \operatorname{st}\left(v_{W_{i}}, T\right) \neq$ $\emptyset$. Thus, the set of vertices $\left\{v_{W_{0}}, \cdots, v_{W_{n}}\right\}$ spans a simplex of $T$. Hence the correspondence $v_{W}, W \in \mathcal{W}$, induces a map $\psi: N(\mathcal{W}) \rightarrow|T|=P$.

Then we shall show that $\psi \circ \eta: Y \rightarrow P$ is a $\operatorname{St}(\mathcal{V})$-map. First we note the following:

$$
d(\varphi, \psi \circ \eta \circ f) \leq \mathcal{T} .
$$

For any $x \in X$, let us take a $W_{0} \in \mathcal{W}$ such that $f(x) \in W_{0}$. Then $\xi_{W_{0}}(f(x))>0$ and $\psi \circ \eta \circ f(x) \in \operatorname{st}\left(v_{W_{0}}, T\right)$. Hence, by (5), $\varphi(x), \psi \circ \eta \circ f(x) \in \operatorname{st}\left(v_{W_{0}}, T\right)$.

Let us fix an arbitrary vertex $v_{0}$ of $T$. Take a given point $y \in(\psi \circ \eta)^{-1}\left(\operatorname{st}\left(v_{0}, T\right)\right)$ and a point $x \in f^{-1}(y)$. Then, by (6), there exists a vertex $v_{x} \in T$ such that $\varphi(x), \psi \circ \eta(y) \in \operatorname{st}\left(v_{x}, T\right)$. Hence when we take $\tilde{U}_{0}, \tilde{U}_{x} \in \tilde{\mathcal{U}}$ such that $\operatorname{st}\left(v_{0}, T\right) \subset$ $\tilde{U}_{0}$ and $\operatorname{st}\left(v_{x}, T\right) \subset \tilde{U}_{x}, \tilde{U}_{x} \cap \tilde{U}_{0} \neq \emptyset$ and $\varphi(x) \in \operatorname{St}\left(\tilde{U}_{0}, \tilde{\mathcal{U}}\right)$. Hence $f^{-1}(y) \subset$ $\varphi^{-1}\left(\operatorname{St}\left(\tilde{U}_{0}, \tilde{\mathcal{U}}\right)\right)$. Therefore, by $(1), f^{-1}(y) \subset r^{-1}\left(V_{0}\right)$ for some $V_{0} \in \mathcal{V}$. Since $d(f, r) \leq \mathcal{V}, y \in f\left(r^{-1}\left(V_{0}\right)\right) \subset S t\left(V_{0}, \mathcal{V}\right)$. Note that choosing $V_{0}$ depends on only the vertex $v_{0}$. It follows that $(\psi \circ \eta)^{-1}\left(s t\left(v_{0}, T\right)\right) \subset S t\left(V_{0}, \mathcal{V}\right)$. Namely $Y$ is $\mathcal{P}$-like.

Next suppose that $Y$ is $\mathcal{P}$-like. For a locally finite open cover $\mathcal{U}$ of $X$ there exists a $\mathcal{U}$-map $f: X \rightarrow Y$ and an open cover $\mathcal{V}$ of $Y$ such that $f^{-1}(\mathcal{V}) \prec \mathcal{U}$. Since $Y$ is $\mathcal{P}$-like, there exist a $P \in \mathcal{P}$ and a $\mathcal{V}$-map $\psi: Y \rightarrow P$. Then the composition $\psi \circ f: X \rightarrow P$ is a $\mathcal{U}$-map. Therefore $X$ is $\mathcal{P}$-like.

By Theorem 2.2 and Proposition 2.1 we can see the following:

Corollary 2.3. Let $r: X \rightarrow Y$ be a refinable map between paracompact spaces. Then $X$ is finitistic if and only if $Y$ is finitistic.

Dranishnikov $[\mathrm{Dr}$ ] gave a remarkable example of a separable metric space $X$ such that $\operatorname{dim}_{\mathbb{Z}} X \leq 4$ but $\operatorname{dim}_{\mathbb{Z}} \beta X=\infty$ and Dydak-Walsh [Dy-W], for any abelian group $G$, constructed a separable metric space $Y$ such that $\operatorname{dim}_{G} Y \leq 3$ but $\operatorname{dim}_{G} \alpha Y>3$ for any compactification $\alpha Y$ of $Y$. In spite of these examples, finitistic spaces, by Rubin-Schapiro theorem, still give a large class of spaces whose Stone-Čech compactifications keep cohomological dimension with respect to any 
finitely generated abelian groups. A current movement of cohomological dimension theory is shifting to a more general notation called extension theory. Namely, for a CW-complex $K$ the extension dimension of a space $X$ is equal or less than $K$, shortly $e$ - $\operatorname{dim} X \leq K$, if every map $f: A \rightarrow K$ of a closed subset $A$ of $X$ to $K$ admits a continuous extension $F: X \rightarrow K$. Corresponding and improved examples of Dranishnikov's and Dydak-Walsh's examples to extension dimension theory were obtained by Levin $[\mathrm{L}$. Here we state a corresponding result with Rubin-Schapiro theorem as follows:

Theorem 2.4 (Dy-M-S , Theorem 4.1). Suppose that $X$ is a finitistic, paracompact space and $K$ is a $C W$-complex of finite type, that is, each skeleton of $K$ is a finite subcomplex. If $e-\operatorname{dim} X \leq K$, then $e-\operatorname{dim} \beta X \leq K$.

Moreover, if $K$ is complete and $e-\operatorname{dim} \beta X \leq K$, then $e-\operatorname{dim} X \leq K$.

We note that for any finitely generated abelian group $G$ and $n \geq 1$ we can have an Eilenberg-MacLane complete complex $K(G, n)$ of finite type.

We state the following fact about Stone-Cech extension of c-refinable maps:

Theorem 2.5 ([Ko1], Theorem 3.1). Let $r: X \rightarrow Y$ be a c-refinable map between normal spaces. Then the extension $\beta f: \beta X \rightarrow \beta Y$ is refinable.

Therefore Theorems 2.4 and 2.5 induce the following result related to extension dimension:

Corollary 2.6. Let $r: X \rightarrow Y$ be a c-refinable map between paracompact spaces. If one of $X$ or $Y$ is finitistic, then another is finitistic, and $e$ - $\operatorname{dim} X \leq K$ for a complete $C W$-complex $K$ of finite type if and only if e- $\operatorname{dim} Y \leq K$.

Remark 1. To investigate extension property of noncompact or nonmetrizable spaces, the notation $\alpha(K)$ introduced by Kuz'minov [Ku] may be useful. The author essentially used the property in [Ko2], and Chigogidze and Valov [C-V] succeeded to characterize extension dimension by using the notation " $\alpha(K)$-like spaces".

\section{Hereditary shape equivalences and finitisticness}

A map between metric spaces is called proper if the preimage of every compact subset is compact, or equivalently the map is closed and has compact fibers. A proper map $f$ from $X$ onto $Y$ is a hereditary shape equivalence if for every closed subset $B$ of $Y$ the restriction $\left.f\right|_{A}: A \rightarrow B, A=f^{-1}(B)$, is a shape equivalence.

Theorem 3.1. Let $f: X \rightarrow Y$ be a hereditary shape equivalence. If $X$ is finitistic, then $Y$ is also finitistic.

For the proof we shall use the following characterization by [H1] and Dy-M-S].

Proposition 3.2. A paracompact space $X$ is finitistic if and only if there exists a compact subspace $K$ of $X$ such that $\operatorname{dim} F<\infty$ for every closed subspace $F$ with $F \cap K=\emptyset$.

Proof of Theorem 3.1. Let $f: X \rightarrow Y$ be a hereditary shape equivalence on a finitistic space $X$. By Proposition 3.2, we take a compact subspace $K$ of $X$ satisfying the desired property. We shall show the compact subspace $f(K)=L$ of $Y$ has the property in Proposition 3.2. 
Let us take a closed subset $F$ of $Y$ with $F \cap L=\emptyset$. Then $f^{-1}(F) \cap K=\emptyset$. Hence $\operatorname{dim} f^{-1}(F)<\infty$. Now the restriction $\left.f\right|_{f^{-1}(F)}: f^{-1}(F) \rightarrow F$ is a hereditary shape equivalence. Therefore $\operatorname{dim} F \leq \operatorname{dim} f^{-1}(F)<\infty$. Thus, $L$ has the required property.

Remark 2. We recall that a proper map $f$ from $X$ onto $Y$ is cell-like if for every $y \in Y, f^{-1}(y)$ has the trivial shape. Namely, the notation of hereditary shape equivalences is a strengthening of cell-like maps. Now let us consider Dranishnikov's separable metric space $X$ in $\overline{\mathrm{Dr}}$ again. Then, by Rubin and Schapiro's cell-like resolution theorem $\mathrm{Ru}-\mathrm{Sc}_{1}$, there can exist a cell-like map from a metric space $Z$ with $\operatorname{dim} Z=\operatorname{dim}_{\mathbb{Z}} X \leq 4$ onto $X$. Thus, a cell-like image of a finitistic space, even a finite-dimensional metric space, is not finitistic.

\section{REFERENCES}

[A] F. Ancel, Proper hereditary shape equivalence property C, Topology and its Appl., 19(1985), 71-74. MR 86g:54026

[B] G. E. Bredon, Introduction to compact transformation groups, Academic Press, 1972, New York. MR 54:1265

[C-V] A. Chigogidze and V. Valov, Extension dimension and refinable maps, preprint(1999).

[De-P] S. Deo and A. R. Pears, A completely finitistic space is finite-dimensional, Bull. London Math. Soc., 17(1985), 49-51. MR 85k:54041

[De-Si ${ }_{1}$ S. Deo and T. B. Singh, On the converse of some theorems about orbit spaces, J. London Math. Soc., 25(1982), 162-170. MR 83k:54039

[De-Si $\left.{ }_{2}\right]$, On certain constructions in finitistic spaces, Internat. J. Math. Math. Sci., 6(1983), 477-482. MR 85c:54058

[De-Si-S] S. Deo, T. B. Singh, and R. A. Shukra, On an extension of localization theorem and generalized Conner conjecture, Trans. Amer. Math. Soc., 269(1982), 395-402. MR 83a:57051

[De-T] S. Deo and H. S. Tripathi, Compact Lie group actions on finitistic spaces, Topology, 21(1982), 393-399. MR 83k:54042

[Di] J. J. Dijkstra, A dimension raising hereditary shape equivalence, Fund. Math., 149(1996), 265-274. MR 97f:54040

[Di-Mo] J. J. Dijkstra and J. Mogilski, Countable dimensionality and dimension raising cell-like maps, 80(1997), 73-79. MR 98i:54017

[Dr] A. N. Dranishnikov, Cohomological dimension is not preserved under Stone-Čech compactification, Comptes Rendus Bulgarian Acad. of Sci., 41(1988), 9-10. MR 90e:55002

[Dy-M-S] J. Dydak, S. N. Mishra and R. A. Shukla, On finitistic spaces, Topology and its Appl., 97(1999), 217-229. MR 2000i:55003

[Dy-W] J. Dydak and J. Walsh, Spaces without cohomological dimension preserving compactifications, Proc. Amer. Math. Soc., 113(1991), 1155-1162. MR 92c:54039

[F-R] Jo Ford and J. Rogers, Jr., Refinable maps, Colloq. Math., 39(1978), 263-269. MR 80d:54009

[G-Ro] D. Garity and D. Rohm, Property $C$, refinable maps and dimension raising maps, Proc. Amer. Math. Soc., 98(1986), 336-340. MR 87i:54077

[H1] Y. Hattori, A note on finitistic spaces, Questions and Answers Gen. Topology, 3(1985), 47-55. MR 86m:54025

[H2] - Finitistic spaces and dimension, Houston J. Math., 25(1999), 687-696.

[Ka] H. Kato, A note on infinite dimension under refinable maps, Proc. Amer. Math. Soc., 88(1983), 177-180. MR 84c:54064

[Ka-Ko] H. Kato and A. Koyama, A role of refinable maps - a survey, Topology Proc., 11(1986), 317-348. MR 89f:54030

[Ko1] A. Koyama, Refinable maps in dimension theory, Topology and its Appl., 17(1984), 247-255. MR 86d:54056

[Ko2] ㄴ Refinable maps in dimension theory II, Bull. Pol. Math. Soc., 42(1994), 255261. CMP 2001:07 
[Ko-Sh] A. Koyama and R. Sher, Approximable dimension and acyclic dimension, Fund. Math., 152(1997), 43-53.

[Ku] V. I. Kuz'minov, Homological dimension theory, Russian Math. Surveys, 23(1968), 1-45. MR 39:2158

[L] M. Levin, Some examples in cohomological dimension theory, preprint(1999).

[Mi] R. Millspaugh, Proper hereditary shape equivalences preserve small weak infinite dimensionality, Proc. Amer. Math. Soc., 90(1984), 1055-1061. MR 91d:54041

[Ru-Sc 1$]$ L. Rubin and P. Schapiro, Cell-like maps onto non-compact spaces of finite cohomological dimension, Topology and its Appl., 27(1987), 221-244. MR 89b:55002

[Ru-Sc $\left.\mathrm{Rc}_{2}\right]$, Compactifications which preserve cohomological dimension, Glasnik Mat., 28(48)(1993), 155-165. MR 95g:54029

[Sw] R. G. Swan, A new method in fixed point theory, Comment. Math. Helv., 34(1960), 1-16. MR 22:5978

Division of Mathematical Sciences, Osaka Kyolku University, Kashiwara, Osaka 5828582, JAPAN

E-mail address: koyama@cc.osaka-kyoiku.ac.jp

Unidad Dovente de Matematicas, E. T. S. I. Montes, Universidad Politíecnica, 28040, MADRID, SPAIN

E-mail address: mam@montes.upm.es 